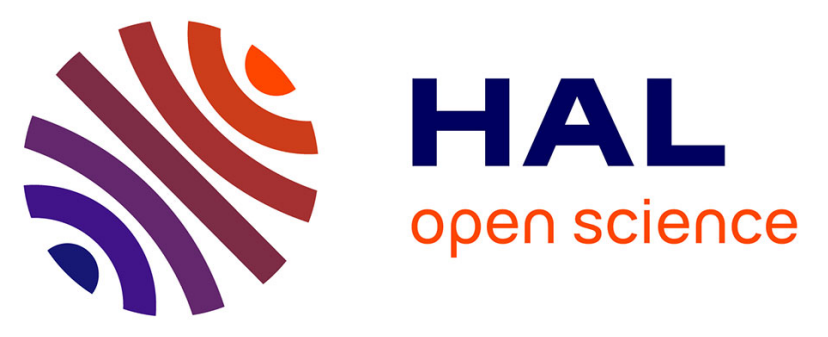

\title{
Scanning Tunneling Microscope-Induced Excitonic Luminescence of a Two-Dimensional Semiconductor
} Delphine Pommier, Rémi Bretel, Luis E Parra López, Florentin Fabre, Andrew Mayne, Elizabeth Boer-Duchemin, Gérald Dujardin, Guillaume Schull, Stéphane Berciaud, Eric Le Moal

\section{To cite this version:}

Delphine Pommier, Rémi Bretel, Luis E Parra López, Florentin Fabre, Andrew Mayne, et al.. Scanning Tunneling Microscope-Induced Excitonic Luminescence of a Two-Dimensional Semiconductor. Physical Review Letters, 2019, 10.1103/PhysRevLett.123.027402 . hal-02183510

\author{
HAL Id: hal-02183510 \\ https://hal.science/hal-02183510
}

Submitted on 15 Jul 2019

HAL is a multi-disciplinary open access archive for the deposit and dissemination of scientific research documents, whether they are published or not. The documents may come from teaching and research institutions in France or abroad, or from public or private research centers.
L'archive ouverte pluridisciplinaire HAL, est destinée au dépôt et à la diffusion de documents scientifiques de niveau recherche, publiés ou non, émanant des établissements d'enseignement et de recherche français ou étrangers, des laboratoires publics ou privés. 


\title{
Scanning Tunneling Microscope-Induced Excitonic Luminescence of a Two-Dimensional Semiconductor
}

\author{
Delphine Pommier, ${ }^{1}$ Rémi Bretel, ${ }^{1}$ Luis E. Parra López, ${ }^{2}$ Florentin Fabre, ${ }^{2}$ Andrew Mayne, ${ }^{1}$ Elizabeth Boer-Duchemin, ${ }^{1}$ \\ Gérald Dujardin, ${ }^{1}$ Guillaume Schull, ${ }^{2}$ Stéphane Berciaud, ${ }^{2}$ and Eric Le Moal ${ }^{1, *}$ \\ ${ }^{1}$ Institut des Sciences Moléculaires d'Orsay, CNRS, Université Paris Sud, Université Paris-Saclay, F-91405 Orsay, France \\ ${ }^{2}$ Institut de Physique et Chimie des Matériaux de Strasbourg, Université de Strasbourg, \\ CNRS, IPCMS, UMR 7504, F-67000 Strasbourg, France
}

(Received 13 December 2018; published 10 July 2019)

\begin{abstract}
The long sought-after goal of locally and spectroscopically probing the excitons of two-dimensional (2D) semiconductors is attained using a scanning tunneling microscope (STM). Excitonic luminescence from monolayer molybdenum diselenide $\left(\mathrm{MoSe}_{2}\right)$ on a transparent conducting substrate is electrically excited in the tunnel junction of an STM under ambient conditions. By comparing the results with photoluminescence measurements, the emission mechanism is identified as the radiative recombination of bright $A$ excitons. STM-induced luminescence is observed at bias voltages as low as those that correspond to the energy of the optical band gap of $\mathrm{MoSe}_{2}$. The proposed excitation mechanism is resonance energy transfer from the tunneling current to the excitons in the semiconductor, i.e., through virtual photon coupling. Additional mechanisms (e.g., charge injection) may come into play at bias voltages that are higher than the electronic band gap. Photon emission quantum efficiencies of up to $10^{-7}$ photons per electron are obtained, despite the lack of any participating plasmons. Our results demonstrate a new technique for investigating the excitonic and optoelectronic properties of 2D semiconductors and their heterostructures at the nanometer scale.
\end{abstract}

DOI: $10.1103 /$ PhysRevLett.123.027402

Two-dimensional (2D) semiconductors such as transition metal dichalcogenides (TMDs; $M X_{2}, M=\mathrm{Mo}, \mathrm{W} ; X=\mathrm{S}$, $\mathrm{Se}, \mathrm{Te})$ exhibit strong light-matter interactions, roomtemperature excitonic effects, and direct band gap emission in the monolayer limit [1-6]. With such assets, TMDs are of enormous interest for photonic and optoelectronic applications in miniaturized photodetectors and lightemitting devices [7-14]. Investigating the optoelectronic response of $2 \mathrm{D}$ semiconductors at the nanometer scale may not only provide fundamental insights into their rich exciton manifold and complex exciton dynamics but also lead to novel quantum devices. In this context, electroluminescence has recently been reported in TMD-based $p-n$ junctions $[9,10,15,16]$, tunnel junctions [14], and quantum-light-emitting diodes [17]. However, in these studies, spatial control over exciton formation was typically limited to the micrometer scale due to the size of the devices. Achieving such control down to the nanoscale is a major challenge that has only been tackled using tipenhanced optical excitation until now $[18,19]$. One particularly promising approach that suppresses the need for optical excitation is the use of a scanning tunneling microscope (STM) because the tunnel current is an extremely local low-energy electrical excitation [20-23]. Nonetheless, STM-induced excitonic luminescence (i.e., the radiative recombination of STM-excited excitons) in a monolayer TMD has not been reported. In previous attempts, the emission of light resulted from the radiative decay of the STM-excited plasmonic modes of the nanocavity formed between a gold tip and a gold surface [24].

In this Letter, we report the first observation of STMinduced excitonic luminescence from a 2D semiconductor (i.e., monolayer $\mathrm{MoSe}_{2}$ ) using a nonplasmonic tip and a nonmetallic transparent conducting substrate. All experiments are carried out under ambient conditions (i.e., in air at room temperature) and the emitted light is collected in transmission using an optical microscope. The observed luminescence is attributed to the radiative recombination of bright $A$ excitons in $\mathrm{MoSe}_{2}$. The photon emission quantum efficiency depends on the bias voltage $V_{s}$. The onset of photon emission is at values of $e V_{s}$ close to the optical band gap of $\mathrm{MoSe}_{2}$, i.e., more than $0.5 \mathrm{eV}$ lower than its electronic band gap. The proposed excitation mechanism is resonance energy transfer from the tunneling current to the excitons in the semiconductor; thus, excitons are created through virtual photon coupling [25]. This fundamentally differs from the charge injection mechanism inferred, for the most part, in previous electroluminescence studies [8-13]. Additional mechanisms (including charge injection) may come into play for bias voltages that are higher than the electronic band gap. Our results demonstrate a new technique for locally probing the elementary 


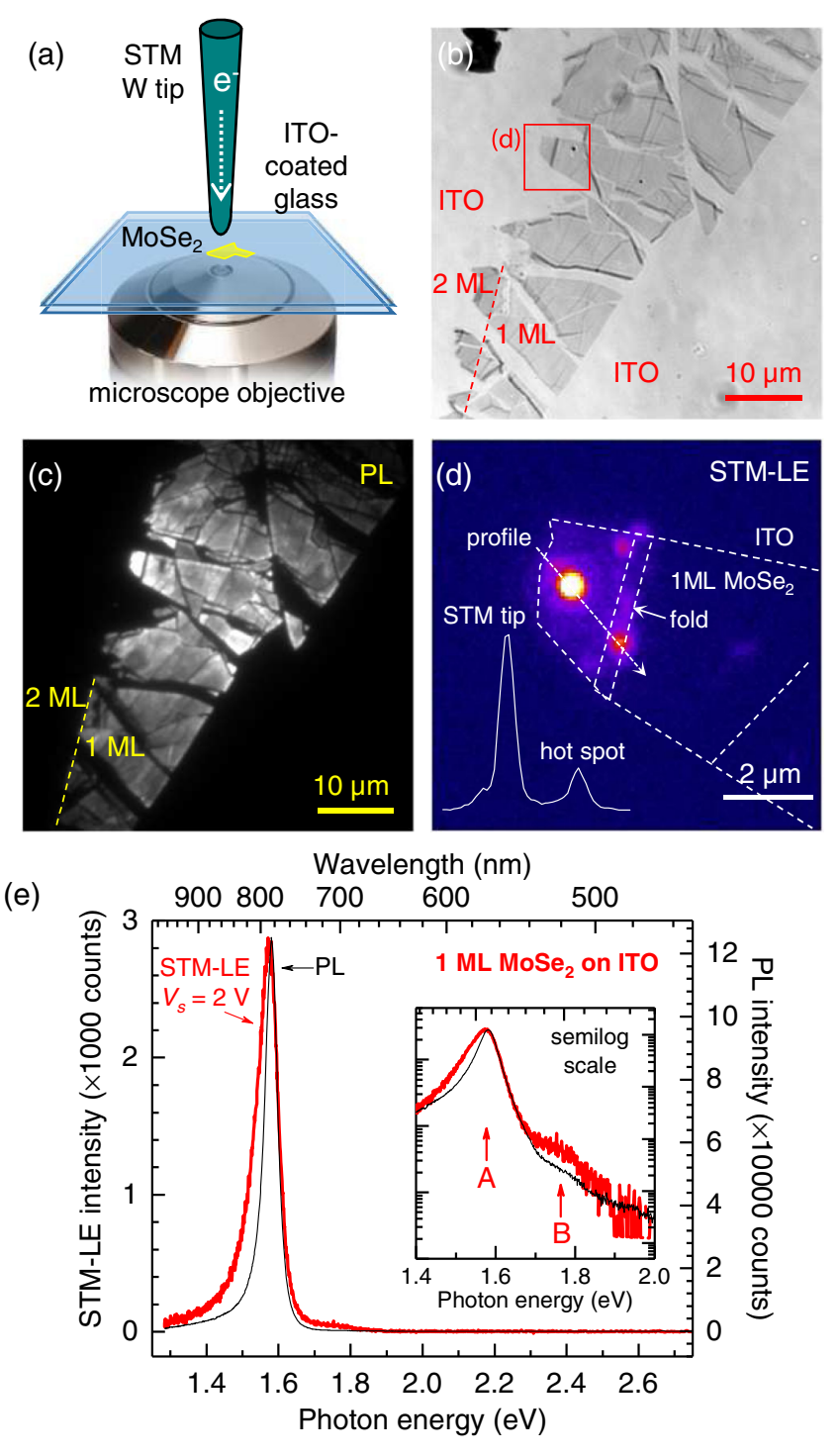

FIG. 1. (a) Schematics of the experiment: the sample is placed between the tungsten tip of an STM and a high-NA microscope objective. The STM tip is grounded and the sample is positively biased. (b) White-light transmission and (c) laser-induced photoluminescence optical microscopy images of the sample, i.e., a thin exfoliated $\mathrm{MoSe}_{2}$ microflake on an ITO-coated glass coverslip. Most of the $\mathrm{MoSe}_{2}$ microflake area has the thickness of a single monolayer (1 ML), except for a small bilayer (2 ML, i.e., twomonolayer) area on the bottom left corner of the image. (d) Realspace optical microscopy image of the STM-induced light from monolayer $\mathrm{MoSe}_{2}$. In the inset of Fig. 1(d) is an intensity profile taken along the dashed line in Fig. 1(d). For STM-LE, $V_{s}=4 \mathrm{~V}$, $I_{t}=5 \mathrm{nA}$, and the acquisition time is $200 \mathrm{~s}$. (e) PL and STM-LE spectra of monolayer $\mathrm{MoSe}_{2}$. PL is laser excitation at $\lambda=465.8 \mathrm{~nm}$, with the longpass filter from $\lambda=491 \mathrm{~nm}$. For STM-LE, $V_{s}=2 \mathrm{~V}$, $I_{t}=10 \mathrm{nA}$, and the acquisition time is $150 \mathrm{~s}$. All spectra are corrected for detection efficiency [30]. In the inset is a semilog plot of the same data, clarifying the $A$ and $B$ exciton contributions.

interactions between electrons, excitons, and photons in 2D semiconductors; such studies are not possible using on-chip electroluminescent devices.
Figure 1(a) shows the experimental setup and the principle of the experiment. An STM head operating in air is mounted on top of an inverted optical microscope. Exfoliated $\mathrm{MoSe}_{2}$ microflakes are deposited onto an indium tin oxide (ITO)coated glass coverslip (ITO thickness of $85 \mathrm{~nm}$ ) using a dry transfer method [26] [see Fig. 1(b)]. The cracks and folds in the flake, visible in Fig. 1(b), result from the transfer process. Luminescence from the sample is electrically induced by the tunneling of electrons from the STM tip (an electrochemically etched tungsten wire) to the sample. For comparison, laser-induced photoluminescence (PL) is also measured using a continuous-wave argon-ion laser emitting at a wavelength of $465.8 \mathrm{~nm}$. Figure 1(c) shows a widefield PL microscopy image of the same area as in Fig. 1(b). Monolayers of $\mathrm{MoSe}_{2}$ are unambiguously distinguished from multilayer specimens due to their bright direct-band gap emission. The emitted light is detected in transmission through the substrate using a high-numerical-aperture (NA $=1.49)$ oil-immersion microscope objective. Optical images are recorded using a cooled CCD camera. The spatial and angular distributions of the emitted light are resolved by imaging the front and back focal planes of the objective on the camera (real-space and Fourier-space imaging), respectively [27-29]. Luminescence spectra are measured using an optical imaging spectrometer. In addition, photon mapping of the STM-induced light emission (STM-LE) is obtained by simultaneously scanning the sample surface with the STM tip and recording the optical signal using an avalanchephotodiode single-photon counting module. The photon emission quantum efficiency is obtained by dividing the optical signal by the measured current and expressing this ratio as a number of emitted photons per tunneling electron.

In Fig. 1(d), the STM tip locally excites a monolayer $\mathrm{MoSe}_{2}$ domain [same area as the one framed in Fig. 1(b)] and the spatial distribution of the resulting light is measured in real space using the optical microscope. An intensity profile taken along a line crossing the location of the STM tip is shown in the inset. Not only is STM-LE emitted from below the STM tip but also from a microscale area around the tip, as defined by the flake boundaries. In addition, localized emission from several "hot spots" (which are 1 to $2 \mu \mathrm{m}$ away from the tip) is detected. Such observations confirm that excitons in monolayer TMDs at room temperature may diffuse micrometers away from the excitation source before radiatively decaying [49-51]. Hot spots at the flake edges and along folds and cracks in the flake may result from longer exciton residence times, or trapping. Below, the emission mechanism of STM-LE is investigated using optical spectroscopy.

The STM-LE and PL spectra from monolayer $\mathrm{MoSe}_{2}$ shown in Fig. 1(e) are similar to each other. At a sample bias of $V_{s}=2 \mathrm{~V}$ and a current set point of $10 \mathrm{nA}$, an STMLE peak at $1.57 \mathrm{eV}(\lambda=788 \mathrm{~nm})$ is observed: the full width of which is $74 \mathrm{meV}$ at half-maximum (FWHM). As shown in the Supplemental Material [30], the lower 
FWHMs are measured at lower currents, i.e., $55 \mathrm{meV}$ at $0.1 \mathrm{nA}$ for the 1.5-3.0 V bias range. Likewise, the PL spectrum of monolayer $\mathrm{MoSe}_{2}$ exhibits an emission peak at $1.58 \mathrm{eV}$ : the FWHM of which is $45 \mathrm{meV}$. These values are typical of the neutral $A$ exciton in $\mathrm{MoSe}_{2}$ at room temperature [52]. The peaks in both spectra in Fig. 1(e) are asymmetric on the low-energy side due to additional contributions from charged excitons (trions) [5] and/or vibronic transitions [53,54]. Such contributions cannot be resolved at room temperature. In addition, the semilog plot shown in Fig. 1(e) clearly shows the existence of a secondary peak blueshifted by $180-190 \mathrm{meV}$ with respect to the $A$ exciton. This shift matches the energy splitting of the valence band due to spin-orbit coupling in monolayer $\mathrm{MoSe}_{2}$ [5]; thus, we ascribe the blueshifted peak to hot luminescence from the $B$ exciton [52]. The match between the PL and STM-LE spectra shown in Fig. 1(e) is evidence that they result from the same emission mechanism. Thus, the dominant emission process of STM-LE from monolayer $\mathrm{MoSe}_{2}$ on ITO is the radiative recombination of $A$ excitons in $\mathrm{MoSe}_{2}$. Other emission mechanisms such as blackbody radiation or radiative electronic transitions between tip and surface states may be ruled out because of the close match of the results to the PL spectrum.

Figure 2 shows a Fourier-space image of the angular distribution of the STM-LE from monolayer $\mathrm{MoSe}_{2}$, which is analyzed using the simple analytical model of an oscillating pointlike electric dipole on an air-glass interface. This model is relevant to describe the far-field emission pattern resulting from the radiative exciton recombination because excitons in monolayer TMDs have a Bohr radius of about $1 \mathrm{~nm}$. The experimental image is juxtaposed with the theoretical emission calculated for two different dipole orientations with respect to the interface [55]. In Fig. 2(b), the dipole is oriented out of plane, i.e., in the direction perpendicular to the sample. In Fig. 2(c), for symmetry reasons, we consider an average over randomly oriented inplane dipoles. As shown in Fig. 2(d), the experimental radial profile clearly differs from that of an out-of-plane electric dipole. In contrast, excellent agreement with the inplane dipole model is found, in particular, within the subcritical angular range and for the angle of maximum emission, without the use of any fitting parameters. These observations confirm that the detected light is not due to a nanocavity mode of the tip-surface junction because such a mode would exhibit an emission pattern similar to that of an out-of plane dipole [56]. These results also confirm that we measure the luminescence of the bright exciton in monolayer $\mathrm{MoSe}_{2}$, which has an in-plane transition dipole (unlike the dark exciton) [57]. Here, bright and dark excitons refer to the spin-allowed and spin-forbidden excitons that result from the conduction band splitting due to spin-orbit coupling, respectively. In molybdenumbased TMDs, bright $A$ excitons have lower energy than their dark counterparts; therefore, PL from monolayer
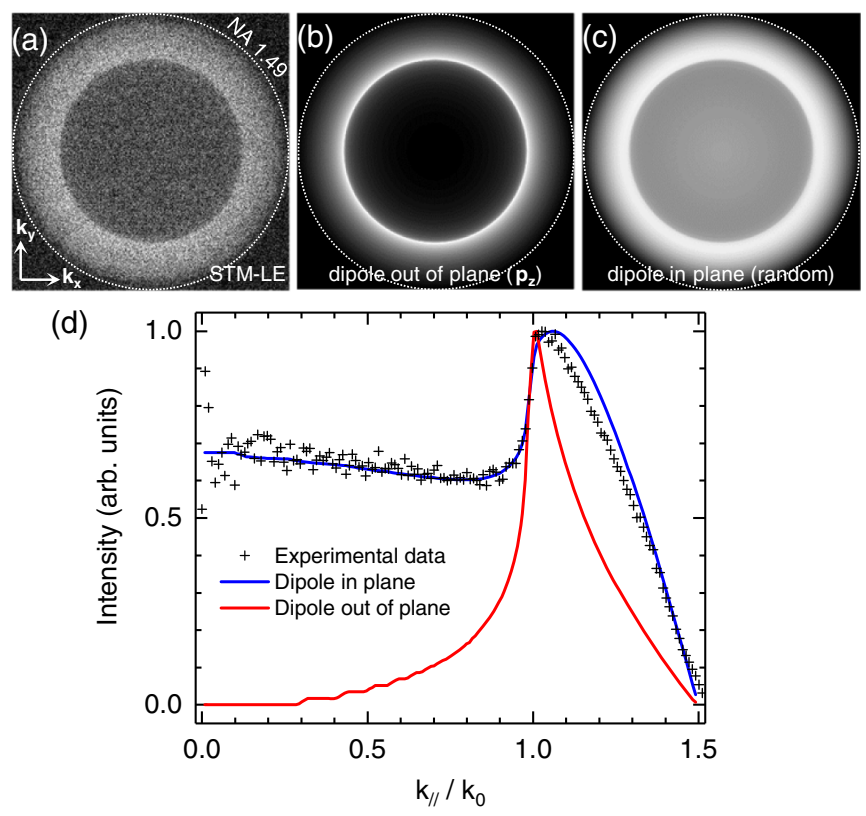

FIG. 2. (a) Fourier-space optical image of the STM-LE measured on monolayer $\mathrm{MoSe}_{2}$. Theoretical emission patterns in Fourier space, calculated for an oscillating pointlike electric dipole on an air-glass interface, for (b) out-of-plane (along $\mathbf{z}$ ) and (c) random in-plane orientations of the dipole. (d) Radial profiles taken from images in Figs. 2(a) to 2(c). The experimental profile is corrected for apodization [59], i.e., multiplied by $\cos \theta$, where $\theta$ is the emission angle in the substrate. $k_{\|} / k_{0}=n \sin \theta$ is the normalized in-plane coordinate in Fourier space, where $k_{0}=\omega / c$ is the photon wave vector modulus and $n$ is the refractive index of the substrate.

$\mathrm{MoSe}_{2}$ is due to bright $A$ excitons [5]. Thus, we conclusively demonstrate that the electrical excitation from the STM induces luminescence from the same excitons as those responsible for the photoluminescence of $\mathrm{MoSe}_{2}$. In our work, no interaction with a plasmonic tip [19] or substrate [58] renders the dark excitons optically active. Neither the substrate nor the STM tip used support surface plasmons within the investigated frequency range (the real parts of the dielectric permittivities of tungsten and ITO are positive).

Figures 3(a) and 3(b) show the STM topography and photon emission quantum efficiency maps of an area where both bilayer and monolayer $\mathrm{MoSe}_{2}$ may be seen. Height and intensity profiles taken along the same lines in Figs. 3(a) and 3(b) are shown in Fig. 3(c). The emission of light is spatially correlated with the presence of monolayer $\mathrm{MoSe}_{2}$ in the tunnel junction and reveals geometrical features of the sample with deep subwavelength lateral resolution, which is not attainable in standard optical microscopy. In particular, the quantum efficiency map exhibits a sharp transition between the bilayer and monolayer areas. This transition occurs over a distance of less than $20 \mathrm{~nm}$. The two areas differ by $0.9 \mathrm{~nm}$ in STM height, i.e., close to the expected thickness of one $\mathrm{MoSe}_{2}$ layer (i.e., $0.65 \mathrm{~nm}$ ). The 


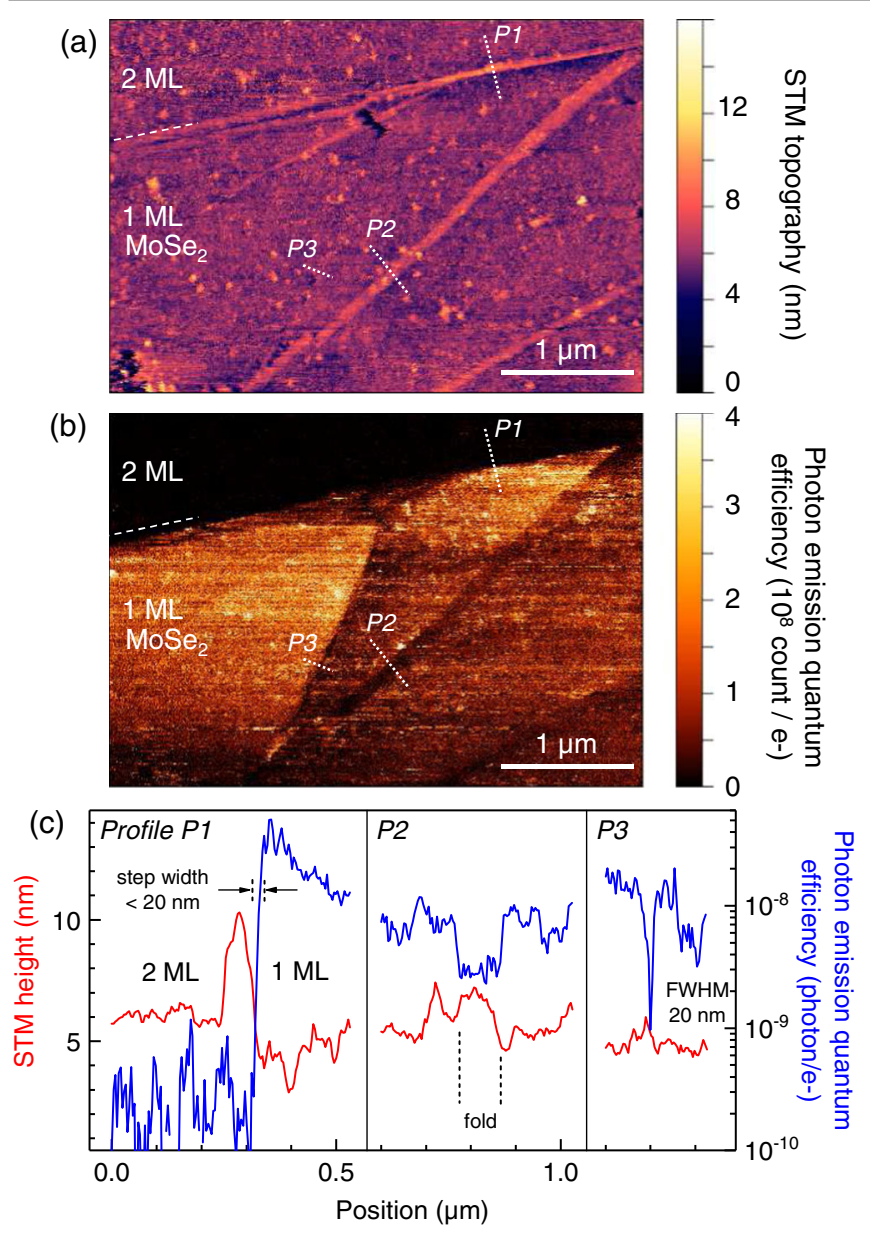

FIG. 3. (a) STM topography of an area featuring bilayer and monolayer $\mathrm{MoSe}_{2}$. (b) Photon emission quantum efficiency (i.e., the number of emitted photons per tunneling electron) obtained from the ratio of the STM-LE photon map and STM current simultaneously recorded with the STM topography shown in Fig. 3(a). (c) Profiles taken along the same lines in Figs. 3(a) and 3(b). STM parameters are $V_{s}=4 \mathrm{~V}$ and $I_{t}=10 \mathrm{nA}$.

photon emission quantum efficiencies measured on the two areas differ from each other by two orders of magnitude. Thus, monolayer vs bilayer $\mathrm{MoSe}_{2}$ may be identified without ambiguity. Furthermore, the imaging of nanoscale defects is demonstrated, e.g., on folds that form in monolayer $\mathrm{MoSe}_{2}$ flakes due to the deposition process, and which may extend over micrometers. As shown in Fig. 3(a), such folds are $1.7 \pm 0.1 \mathrm{~nm}$ in height (as compared to the rest of the monolayer) and 10 to $100 \mathrm{~nm}$ in lateral width. In standard optical microscopy, such structures appear as one-dimensional objects. In contrast, the map shown in Fig. 3(b) reveals lateral variations of STM-LE on a 10 to $20 \mathrm{~nm}$ scale, with a decrease of the photon emission quantum efficiency on the folds. Moreover, STM-LE makes it possible to uncover geometrical features such as a "domain boundary" in Fig. 3(b) (see profile P3); i.e., presumably, a nanometerwide crack in the monolayer, which is almost undetectable in the STM topography image. This domain boundary has a strong effect on the photon emission quantum efficiency, which drops by a factor of about five over a distance of 10 to $20 \mathrm{~nm}$.

In order to elucidate the excitation mechanism through which excitons are electrically created in $\mathrm{MoSe}_{2}$, we examine the dependence of the STM-LE on the bias voltage. To this purpose, a series of STM-LE spectra from monolayer $\mathrm{MoSe}_{2}$ is measured at current set points of 0.1 and $10 \mathrm{nA}$, as well as sample biases $V_{s}$ varying within the 1.3-4.0 $\mathrm{V}$ range [30]. The photon emission quantum efficiency is obtained from the area beneath the STMLE peaks and the simultaneously measured tunnel current integrated over the acquisition time. Figure 4 shows a plot of the photon emission quantum efficiency vs bias voltage. At $V_{s}=4 \mathrm{~V}$, about $3 \times 10^{-8}$ photons per electron are detected, which correspond to $10^{-7}$ photons per electron after correction for the detection efficiency (i.e., $31 \%$ at $\lambda_{0}=783 \mathrm{~nm}$ ). At both current set points of 0.1 and $10 \mathrm{nA}$, the onset of luminescence occurs at electron energies close to $1.5 \mathrm{eV}$, matching the optical gap of monolayer $\mathrm{MoSe}_{2}$ $[5,60,61]$. The latter observation is a strong indication that the luminescence is excited by the resonance energy transfer from the tunneling current to the excitons, i.e., through virtual photon coupling. A classical picture of the proposed excitation mechanism is that the tunnel current generates an oscillating electric dipole in the tip-surface gap that transfers energy to the semiconductor via near-field electromagnetic coupling. In a diodelike excitation mechanism, however, where electrons and holes are injected in the bands of the semiconductor, the onset of luminescence would occur at an electron energy higher than the "free particle" electronic band gap of monolayer $\mathrm{MoSe}_{2}$ [23].

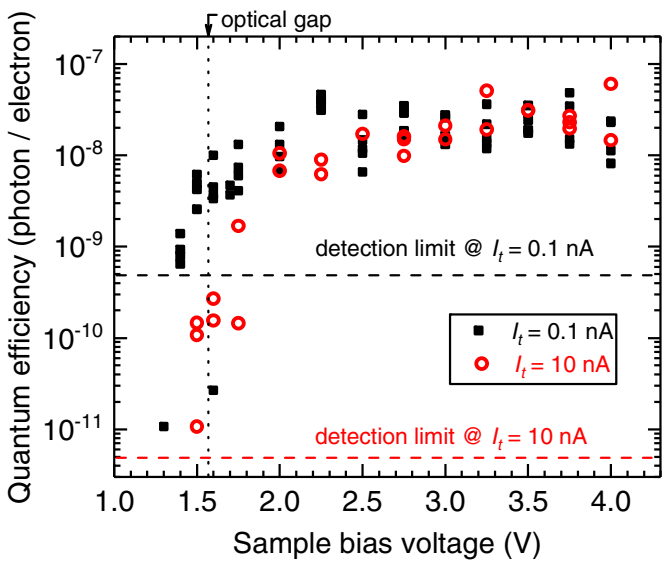

FIG. 4. Photon emission quantum efficiency (i.e., the number of emitted photons per tunneling electron) vs the sample bias voltage, which are obtained from STM-induced luminescence spectra of monolayer $\mathrm{MoSe}_{2}$ measured at current set points of 0.1 and $10 \mathrm{nA}$ (see spectra in [30]). The detection limit is defined from the standard deviation of the dark current of the CCD camera and the STM set point current. A vertical dotted line indicates the optical gap energy of monolayer $\mathrm{MoSe}_{2}$. 
The energy of the electronic band gap of monolayer $\mathrm{MoSe}_{2}$ exceeds that of its optical band gap by more than $0.5 \mathrm{eV}$ $[5,60,61]$; thus, the two possible excitation mechanisms are distinguished by determining the lowest bias voltage at which luminescence is detected. Nevertheless, at sufficiently high electron energies $e V_{s}$ above the electronic band gap, the two processes (energy transfer and charge injection) may be present (see the Supplemental Material [30] for further details).

Finally, in the present work, we describe the excitation mechanism in terms of virtual photon coupling because energy transfer from the tunnel current to the excitons occurs without the participation of an intermediary optical mode such as a localized surface plasmon polariton [62]. In a different context (i.e., STM electroluminescence of single molecules), a comparable excitation mechanism has recently been reported in terms of inelastic electronmolecule scattering [63].

In conclusion, the local electrically excited excitonic luminescence from a 2D semiconductor in the tunnel junction of an STM has been detected using a nonmetallic, transparent substrate (ITO-coated glass) and a nonplasmonic (tungsten) tip combined with high-numerical-aperture collection optics (i.e., an oil-immersion microscope objective). In particular, the emitted light is unambiguously identified as the radiative decay of bright $A$ excitons in monolayer $\mathrm{MoSe}_{2}$. This origin is fundamentally different from that reported in a previous STM study of gold-supported monolayer $\mathrm{MoS}_{2}$ [24], where the emitted light was from plasmons. Our approach, based on the absence of coupling to a plasmonic tip or nanocavity, makes it possible to probe locally the "native" excitonic properties of a 2D semiconductor. In addition, in our study, the onset of luminescence at electron energies as low as the optical band gap of monolayer $\mathrm{MoSe}_{2}$ is consistent with a resonance energy transfer mechanism from the tunneling current to excitons in the 2D semiconductor. Real-space optical microscopy images reveal that the excited excitons may diffuse over micrometers from the STM tip before radiatively decaying; i.e., light may be emitted from a microscale area. Nonetheless, the exciton creation process itself is highly localized, which yields a lateral resolution on the order of 10 to $20 \mathrm{~nm}$ in photon maps recorded upon raster scanning the STM tip across the sample. The present work demonstrates the use of STM-LE as a tool to study exciton dynamics and the optoelectronics properties of 2D semiconductors at the nanometer scale. In particular, with this technique, such investigations may be carried out in air under ambient conditions, i.e., in the environment where devices based on these materials will operate. We anticipate that such investigations will become crucial in the studies of van der Waals heterostructures [64-66], TMD superlattices, and quantumwell-based optoelectronic devices [67-69]. They will also be of importance for the local control of exciton energies and dynamics in strained TMDs [70,71], as well as the localization of excitons in pointlike defects and their nonclassical emission properties [17].

This work is supported by public grants from the French National Research Agency (No. H2DH ANR-15CE24-0016, No. Intelplan ANR-15-CE24-0020, and No. M-Exc-ICO ANR-16-CE24-0003) and Labex NIE (No. ANR-11-LABX-0058-NIE). We thank C. Genet, S. Azzini, and E. Lorchat for insightful discussions, as well as the STnano clean room staff for technical support. S. B. acknowledges support as a Member of the Institut Universitaire de France. This project has received funding from the European Research Council (ERC) under the European Union's Horizon 2020 research and innovation program (Grant No 771850).

*eric.le-moal@u-psud.fr

[1] K. F. Mak, C. Lee, J. Hone, J. Shan, and T. F. Heinz, Phys. Rev. Lett. 105, 136805 (2010).

[2] A. Splendiani, L. Sun, Y. Zhang, T. Li, J. Kim, C.-Y. Chim, G. Galli, and F. Wang, Nano Lett. 10, 1271 (2010).

[3] K. F. Mak and J. Shan, Nat. Photonics 10, 216 (2016).

[4] V. W. Brar, A. R. Koltonow, and J. Huang, ACS Photonics 4, 407 (2017).

[5] G. Wang, A. Chernikov, M. M. Glazov, T. F. Heinz, X. Marie, T. Amand, and B. Urbaszek, Rev. Mod. Phys. 90, 021001 (2018).

[6] V. W. Brar, M. C. Sherrott, and D. Jariwala, Chem. Soc. Rev. 47, 6824 (2018).

[7] A. K. Geim and I. V. Grigorieva, Nature (London) 499, 419 (2013)

[8] R. Cheng, D. Li, H. Zhou, C. Wang, A. Yin, S. Jiang, Y. Liu, Y. Chen, Y. Huang, and X. Duan, Nano Lett. 14, 5590 (2014).

[9] M. M. Furchi, A. Pospischil, F. Libisch, J. Burgdörfer, and T. Mueller, Nano Lett. 14, 4785 (2014).

[10] J. S. Ross, P. Klement, A. M. Jones, N. J. Ghimire, J. Yan, D. G. Mandrus, T. Taniguchi, K. Watanabe, K. Kitamura, W. Yao, D. H. Cobden, and X. Xu, Nat. Nanotechnol. 9, 268 (2014).

[11] G. Clark, J. R. Schaibley, J. Ross, T. Taniguchi, K. Watanabe, J. R. Hendrickson, S. Mou, W. Yao, and X. Xu, Nano Lett. 16, 3944 (2016).

[12] D. Jariwala, S. L. Howell, K.-S. Chen, J. Kang, V. K. Sangwan, S. A. Filippone, R. Turrisi, T. J. Marks, L. J. Lauhon, and M. C. Hersam, Nano Lett. 16, 497 (2016).

[13] C.-H. Liu, G. Clark, T. Fryett, S. Wu, J. Zheng, F. Hatami, X. Xu, and A. Majumdar, Nano Lett. 17, 200 (2017).

[14] J. Binder, F. Withers, M. R. Molas, C. Faugeras, K. Nogajewski, K. Watanabe, T. Taniguchi, A. Kozikov, A. K. Geim, K. S. Novoselov, and M. Potemski, Nano Lett. 17, 1425 (2017).

[15] Y. J. Zhang, T. Oka, R. Suzuki, J. T. Ye, and Y. Iwasa, Science 344, 725 (2014).

[16] B. W. H. Baugher, H. O. H. Churchill, Y. Yang, and P. Jarillo-Herrero, Nat. Nanotechnol. 9, 262 (2014).

[17] C. Palacios-Berraquero, M. Barbone, D. M. Kara, X. Chen, I. Goykhman, D. Yoon, A. K. Ott, J. Beitner, K. Watanabe, 
T. Taniguchi, A. C. Ferrari, and M. Atatüre, Nat. Commun. 7, 12978 (2016).

[18] K.-D. Park, O. Khatib, V. Kravtsov, G. Clark, X. Xu, and M. B. Raschke, Nano Lett. 16, 2621 (2016).

[19] K.-D. Park, T. Jiang, G. Clark, X. Xu, and M. B. Raschke, Nat. Nanotechnol. 13, 59 (2018).

[20] X. H. Qiu, G. V. Nazin, and W. Ho, Science 299, 542 (2003).

[21] Y. Zhang, Y. Luo, Y. Zhang, Y.-J. Yu, Y.-M. Kuang, L. Zhang, Q.-S. Meng, Y. Luo, J.-L. Yang, Z.-C. Dong, and J. G. Hou, Nature (London) 531, 623 (2016).

[22] H. Imada, K. Miwa, M. Imai-Imada, S. Kawahara, K. Kimura, and Y. Kim, Nature (London) 538, 364 (2016).

[23] B. Doppagne, M. C. Chong, E. Lorchat, S. Berciaud, M. Romeo, H. Bulou, A. Boeglin, F. Scheurer, and G. Schull, Phys. Rev. Lett. 118, 127401 (2017).

[24] N. Krane, C. Lotze, J. M. Läger, G. Reecht, and K. J. Franke, Nano Lett. 16, 5163 (2016).

[25] D. L. Andrews and D. S. Bradshaw, Ann. Phys. (Amsterdam) 526, 173 (2014).

[26] A. Castellanos-Gomez, M. Buscema, R. Molenaar, V. Singh, L. Janssen, H. S. J. v. d. Zant, and G. A. Steele, 2D Mater. 1, 011002 (2014).

[27] M. A. Lieb, J. M. Zavislan, and L. Novotny, J. Opt. Soc. Am. B 21, 1210 (2004).

[28] T. Wang, E. Boer-Duchemin, Y. Zhang, G. Comtet, and G. Dujardin, Nanotechnology 22, 175201 (2011).

[29] E. Le Moal, S. Marguet, B. Rogez, S. Mukherjee, P. Dos Santos, E. Boer-Duchemin, G. Comtet, and G. Dujardin, Nano Lett. 13, 4198 (2013).

[30] See Supplemental Material at http://link.aps.org/ supplemental/10.1103/PhysRevLett.123.027402 for additional spectra and experimental details, more data analysis, and detail of the excitation mechanism, which includes Refs. [31-48].

[31] F. Matino, L. Persano, V. Arima, D. Pisignano, R. I. R. Blyth, R. Cingolani, and R. Rinaldi, Phys. Rev. B 72, 085437 (2005).

[32] S. Dasgupta, M. Lukas, K. Dössel, R. Kruk, and H. Hahn, Phys. Rev. B 80, 085425 (2009).

[33] P. D. C. King, T. D. Veal, F. Fuchs, C. Y. Wang, D. J. Payne, A. Bourlange, H. Zhang, G. R. Bell, V. Cimalla, O. Ambacher, R. G. Egdell, F. Bechstedt, and C. F. McConville, Phys. Rev. B 79, 205211 (2009).

[34] C. C. Neacsu, G. A. Steudle, and M. B. Raschke, Appl. Phys. B 80, 295 (2005).

[35] R. Pechou, R. Coratger, F. Ajustron, and J. Beauvillain, Appl. Phys. Lett. 72, 671 (1998).

[36] G. Schull, N. Néel, P. Johansson, and R. Berndt, Phys. Rev. Lett. 102, 057401 (2009).

[37] N. L. Schneider, P. Johansson, and R. Berndt, Phys. Rev. B 87, 045409 (2013).

[38] F. Xu, C. Holmqvist, and W. Belzig, Phys. Rev. Lett. 113, 066801 (2014).

[39] A. M. Jones, H. Yu, J. R. Schaibley, J. Yan, D. G. Mandrus, T. Taniguchi, K. Watanabe, H. Dery, W. Yao, and X. Xu, Nat. Phys. 12, 323 (2016).

[40] J. Jadczak, L. Bryja, J. Kutrowska-Girzycka, P. Kapuściński, M. Bieniek, Y.-S. Huang, and P. Hawrylak, Nat. Commun. 10, 107 (2019).
[41] M. Manca, M. M. Glazov, C. Robert, F. Cadiz, T. Taniguchi, K. Watanabe, E. Courtade, T. Amand, P. Renucci, X. Marie, G. Wang, and B. Urbaszek, Nat. Commun. 8, 14927 (2017).

[42] B. Han, C. Robert, E. Courtade, M. Manca, S. Shree, T. Amand, P. Renucci, T. Taniguchi, K. Watanabe, X. Marie, L. E. Golub, M. M. Glazov, and B. Urbaszek, Phys. Rev. X 8, 031073 (2018).

[43] J. Lambe and S. L. McCarthy, Phys. Rev. Lett. 37, 923 (1976).

[44] J. K. Gimzewski, J. K. Sass, R. R. Schlitter, and J. Schott, Europhys. Lett. 8, 435 (1989).

[45] R. Berndt, J. K. Gimzewski, and P. Johansson, Phys. Rev. Lett. 67, 3796 (1991).

[46] P. Johansson, Phys. Rev. B 58, 10823 (1998).

[47] A. Muñoz Losa, C. Curutchet, B. P. Krueger, L. R. Hartsell, and B. Mennucci, Biophys. J. 96, 4779 (2009).

[48] Z. C. Dong, X. L. Zhang, H. Y. Gao, Y. Luo, C. Zhang, L. G. Chen, R. Zhang, X. Tao, Y. Zhang, J. L. Yang, and J. G. Hou, Nat. Photonics 4, 50 (2010).

[49] S. Mouri, Y. Miyauchi, M. Toh, W. Zhao, G. Eda, and K. Matsuda, Phys. Rev. B 90, 155449 (2014).

[50] T. Kato and T. Kaneko, ACS Nano 10, 9687 (2016).

[51] M. Kulig, J. Zipfel, P. Nagler, S. Blanter, C. Schüller, T. Korn, N. Paradiso, M. M. Glazov, and A. Chernikov, Phys. Rev. Lett. 120, 207401 (2018).

[52] G. Froehlicher, E. Lorchat, and S. Berciaud, Phys. Rev. X 8, 011007 (2018).

[53] D. Christiansen, M. Selig, G. Berghäuser, R. Schmidt, I. Niehues, R. Schneider, A. Arora, S. M. de Vasconcellos, R. Bratschitsch, E. Malic, and A. Knorr, Phys. Rev. Lett. 119, 187402 (2017).

[54] I. Niehues, R. Schmidt, M. Drüppel, P. Marauhn, D. Christiansen, M. Selig, G. Berghäuser, D. Wigger, R. Schneider, L. Braasch, R. Koch, A. Castellanos-Gomez, T. Kuhn, A. Knorr, E. Malic, M. Rohlfing, S. Michaelis de Vasconcellos, and R. Bratschitsch, Nano Lett. 18, 1751 (2018).

[55] L. Novotny and B. Hecht, Principles of Nano-Optics (Cambridge University Press, Cambridge, England, 2006).

[56] E. Le Moal, S. Marguet, D. Canneson, B. Rogez, E. BoerDuchemin, G. Dujardin, T. V. Teperik, D.-C. Marinica, and A. G. Borisov, Phys. Rev. B 93, 035418 (2016).

[57] J. A. Schuller, S. Karaveli, T. Schiros, K. He, S. Yang, I. Kymissis, J. Shan, and R. Zia, Nat. Nanotechnol. 8, 271 (2013).

[58] Y. Zhou, G. Scuri, D. S. Wild, A. A. High, A. Dibos, L. A. Jauregui, C. Shu, K. De Greve, K. Pistunova, A. Y. Joe, T. Taniguchi, K. Watanabe, P. Kim, M. D. Lukin, and H. Park, Nat. Nanotechnol. 12, 856 (2017).

[59] J. A. Kurvits, M. Jiang, and R. Zia, J. Opt. Soc. Am. A 32, 2082 (2015).

[60] M. M. Ugeda, A. J. Bradley, S.-F. Shi, F. H. da Jornada, Y. Zhang, D. Y. Qiu, W. Ruan, S.-K. Mo, Z. Hussain, Z.-X. Shen, F. Wang, S. G. Louie, and M. F. Crommie, Nat. Mater. 13, 1091 (2014).

[61] M. Koperski, M. R. Molas, A. Arora, K. Nogajewski, A. O. Slobodeniuk, C. Faugeras, and M. Potemski, Nanophotonics 6, 1289 (2017). 
[62] J. Kröger, B. Doppagne, F. Scheurer, and G. Schull, Nano Lett. 18, 3407 (2018).

[63] G. Chen, Y. Luo, H. Gao, J. Jiang, Y. Yu, L. Zhang, Y. Zhang, X. Li, Z. Zhang, and Z. Dong, Phys. Rev. Lett. 122, 177401 (2019).

[64] M. Massicotte, P. Schmidt, F. Vialla, K. Watanabe, T. Taniguchi, K. J. Tielrooij, and F. H. L. Koppens, Nat. Commun. 7, 12174 (2016).

[65] P. Ma, N. Flöry, Y. Salamin, B. Baeuerle, A. Emboras, A. Josten, T. Taniguchi, K. Watanabe, L. Novotny, and J. Leuthold, ACS Photonics 5, 1846 (2018).

[66] M. Parzefall, A. Szabó, T. Taniguchi, K. Watanabe, M. Luisier, and L. Novotny, Nat. Commun. 10, 292 (2019).
[67] X. Su, R. Zhang, C. Guo, M. Guo, and Z. Ren, Phys. Chem. Chem. Phys. 16, 1393 (2014).

[68] A. Azizi, Y. Wang, Z. Lin, K. Wang, A. L. Elias, M. Terrones, V.H. Crespi, and N. Alem, Nano Lett. 16, 6982 (2016).

[69] S. Xie, L. Tu, Y. Han, L. Huang, K. Kang, K. U. Lao, P. Poddar, C. Park, D. A. Muller, R. A. DiStasio, and J. Park, Science 359, 1131 (2018).

[70] R. K. Defo, S. Fang, S. N. Shirodkar, G. A. Tritsaris, A. Dimoulas, and E. Kaxiras, Phys. Rev. B 94, 155310 (2016).

[71] D. F. Cordovilla Leon, Z. Li, S. W. Jang, C.-H. Cheng, and P. B. Deotare, Appl. Phys. Lett. 113, 252101 (2018). 